Sir,

\section{Response to Johnson et al}

We thank Johnson et al ${ }^{1}$ for their interest in our study. ${ }^{2}$ In response to their statements.

'Notwithstanding concern that the community service did not use clinicians with specialist training and a glaucoma qualification awarded by the appropriate professional body as demanded by NICE'

This study predated NICE guidance; however, all the optometrists received extensive training using a competency-based model very similar to that which is currently used by the College of Optometrists. The accreditation in the scheme included a series of written papers to test knowledge of glaucoma and skill-based practical assessments together with an assessment of clinical decision making using a structured oral examination and unseen case records. All optometrists carried out a clinical placement in a glaucoma clinic consisting of a minimum of 24 sessions.

'We would like to suggest why the model does not agree with reality.'

The aim of our research was to investigate a model of care provision. We set the model up and allowed it to run first before investigating the costs and other aspects in detail. As far as we are aware, this is only the second study to undertake a formal cost analysis under rigorous research conditions. What we found is the reality in our instance, which is the whole point of undertaking the research.

'The community model was based on the appointment structure of sight tests that involve refraction'

The community model involved no refraction.

'only 11 patients being seen per day. In contrast, it is typical for optometrists working in hospital glaucoma clinics to have a daily caseload of 20 patients. We have been told by an author of the study that this reflected the inclusion of 'non-stable' cases (Professor Lawrenson, personal communication), but remain unconvinced that such a large reduction in volume is needed.'

The numbers seen per clinician in any single setting is dependent on many factors and case mix is certainly one major contributor. We are aware that this number in our study seems low. The number was not our derivation but an optometrist-driven derivation of the numbers they felt confident in managing. We addressed this issue in more detail in the discussion by undertaking a sensitivity analysis, which shows that even at numbers suggested by Johnson et al ${ }^{1}$ our model would still have not been cost effective. Clinical safety and record keeping may be potentially affected by numbers. We have investigated these outcomes and will be reporting on them.

'The opportunity cost to optometrists relative to their usual business of providing sight tests and selling spectacles was used as a surrogate for the cost of providing glaucoma services. A wide range of values have been reported for this figure, which is not unexpected given the large variation in overheads and retail income. Recently, in a joint publication by the main optical bodies it was estimated that the daily cost of running a practice was $£ 910-1225,3$ which is less than the $£ 1601.81$ suggested by optometrists involved in this study. The inflated opportunity cost in this study may reflect the relatively high rental rates and retail income in London, but their disagreement with other reports indicates that they may not be applicable to other regions.'

It is given that overhead costs will vary from region to region. We took some considerable effort in arriving at our overall costs and attempted to explain the methodology. All the optometrists completed the costs individually and there was then a consensus meeting where the submissions were jointly considered before arriving at our published figure. Practice overhead costs are relatively easy for practices to compute and did not disagree widely from the figures quoted by Johnson et al. The opportunity cost is the 'value added' or 'value subtracted' from the business as a result of the clinic being run within the practice. There was uniform agreement that the clinics resulted in income loss. The consensus meeting costed this at half a receptionist salary for time to explain to other 'customers' why the waiting room was so full and what was going on. This is why the figure is larger.

'Insufficient details were given in the paper to allow for an opinion on the validity of cost estimates of the hospital-based glaucoma service, but of more relevance is the fact that hospital services in the UK use a fixed tariff system and so from the view of the funding body no estimate is needed.'

The fixed tariff system is a government-derived figure and certainly could be used but is an extremely crude tool compared with our published figures. We have provided, for the first time, the real costs derived by rigorous micro-costing. This demands time analysis of individuals multiplied by their real salaries, actual supply costs of expendables, actual ground rents, actual electrical costs and so on. An NHS hospital is there for the patients and has no other 'business', hence, the opportunity cost is neutral.

'The discrepancy between the study results and our experiences shows that the cost of a particular model of community-based glaucoma services cannot be generalized to all community-based glaucoma services. It is also important to appreciate that optometrists working in community can improve accessibility and increase capacity of glaucoma services, which is relevant in the context of the typically elderly glaucoma population and increasing appointment delays that have already led to avoidable sight loss.'

The above statements are, of course, correct. What we believe our study adds is a second formal cost analysis of a model of glaucoma care to enable informed discussion and progress.

\section{Conflict of interest}

The authors declare no conflict of interest. 


\section{References}

1 Johnson M, Jones A, Tickner J. Response to 'An economic comparison of hospital-based and community-based glaucoma clinics'. Eye 2012; 26(11): 1493.

2 Sharma A, Jofre-Bonet M, Panca M, Lawrenson JG, Murdoch I. An economic comparison of hospital-based and community-based glaucoma clinics. Eye 2012; 26: 967-971.

3 What community-based optical practices can offer healthcare commissioners and patients. http://www.aop.org.uk/ uploads/uploaded_files/napc_joint_proposal_ april_2008.pdf (accessed 2 June 2012).

A Sharma', M Jofre-Bonet² ${ }^{2}$ M Panca², J Lawrenson ${ }^{3}$ and I Murdoch ${ }^{1}$

${ }^{1}$ Department of Genetics, Institute of Ophthalmology, London, UK

${ }^{2}$ Department of Economics, City University London, London, UK

${ }^{3}$ Department of Optometry and Visual Science,

City University London, London, UK

E-mail: anu1478@hotmail.co.uk

Eye (2012) 26, 1494-1495; doi:10.1038/eye.2012.176; published online 24 August 2012

\section{Sir,}

\section{An unexpected complication of behavioural vision therapy}

Behavioural vision therapy is a branch of optometry that attempts to improve visual efficiency through the prescription of treatments such as hand-eye co-ordination activities. Here we report an unfortunate adverse event associated with such therapy.

\section{Case report}

A 43-year-old female, presented to the Gold Coast Hospital, Queensland suffering a large, vertical, ragged, and shelfed corneal laceration, which occurred while assisting her son to perform a behavioural vision therapy exercise. The vision therapy task belonged to a group of vision therapies known as 'visual-motor integration' activities, promoted for the treatment of reading dysfunction. To perform the exercise, the patient is instructed to attach a tennis ball to a string (via a hook previously screwed into the tennis ball) and hang the string from the ceiling. The task for the patient's son was to gently strike the ball with a wooden rolling pin, which is ringed by coloured bands. Using different sequences of colours, the ball is hit towards a target held by his mother. During the activity, the tennis ball came free of the screw, and the screw (still attached to the string) recoiled against the patient's right eye.

Surgical repair was undertaken and at the most recent review, 1 month following the initial injury, pinhole visual acuity was $\mathrm{R}$ 6/18.

\section{Comment}

Behavioural optometry is concerned with visual dysfunction that proponents believe can exist despite the presence of good visual acuity, no refractive error or ocular disease, normal accommodation, normal binocular vision, and normal ocular motility. ${ }^{1}$ Examples of behavioural vision therapy tasks include hand-eye co-ordination activities, coloured overlay lenses, trampolines, or balance boards. A recent critical appraisal of vision therapy by Barrett ${ }^{2}$ found no significant evidence to support the vast majority of behavioural management approaches advocated by behavioural optometrists, a similar finding to an earlier review by Jennings, $2000 .^{3}$

Our patient was unfortunate to suffer a severe penetrating eye injury while helping her son perform a task of uncertain benefit to his visual development. Any equipment used for such exercises should be as safe as possible to minimise the potential harms of therapy. The use of exercises in which balls are propelled at against a flat surface should be certainly be appraised in this light.

\section{Conflict of interest}

The authors declare no conflict of interest.

\section{References}

1 Scheiman M. Understanding \& Managing Vision Deficits: A guide for Occupational Therapists. 2nd edn. Slack Inc: Philadelphia, 2002

2 Barrett B. A critical evaluation of the evidence supporting the practice of behavioural vision therapy. Ophthalmic Physiol Opt 2009; 29: 4-25.

3 Jennings J. Behavioural optometry - a critical review. Optom Pract 2000; 1: 67-78.

\section{B Drury and F Imrie}

Department of Ophthalmology, Gold Coast Hospital, Southport, Queensland, Australia

E-mail: brett.drury1@gmail.com

Eye (2012) 26, 1495; doi:10.1038/eye.2012.157;

published online 17 August 2012

Sir,

Snailtrack corneal changes following subconjunctival injection of 5-fluorouracil

We report snailtrack-like corneal changes occurring after subconjunctival injection of 5-flurouracil (5-FU) around a trabeculectomy bleb.

\section{Case report}

A 50-year-old male with advanced glaucoma underwent an uncomplicated right-sided trabeculectomy, 